\title{
ASYMPTOTIC RESULTS FOR THE ABSORPTION TIMES OF RANDOM WALKS WITH A BARRIER
}

UDC 519.21

\author{
PAVLO NEGADAĬLOV
}

\begin{abstract}
A sequence $R_{k}^{(n)}:=R_{k-1}^{(n)}+\xi_{k} 1_{\left\{R_{k-1}^{(n)}+\xi_{k}<n\right\}}, k \in \mathbf{N}, R_{0}^{(n)}:=0$, is called a random walk with a barrier $n \in \mathbf{N}$, where the $\xi_{k}$ are independent copies of a random variable $\xi$ assuming positive integer values. The asymptotic behavior of the absorption times is studied in the paper for a random walk with a barrier. This behavior depends on the properties of the tail of the distribution of the random variable $\xi$.
\end{abstract}

\section{INTRODUCTION AND MAIN RESULTS}

Let $\xi$ be a random variable with a proper distribution

$$
p_{k}:=\mathrm{P}\{\xi=k\}, \quad k \in \mathbf{N} .
$$

For simplicity, we assume that $p_{1}>0$ in what follows.

For every $n \in \mathbf{N}$, we define the random walk with the barrier $n$ to be

$$
R_{0}^{(n)}:=0, \quad R_{k}^{(n)}:=R_{k-1}^{(n)}+\xi_{k} 1_{\left\{R_{k-1}^{(n)}+\xi_{k}<n\right\}}, \quad k \in \mathbf{N},
$$

where $\left\{\xi_{k}: k \in \mathbf{N}\right\}$ are independent copies of the random variables $\xi$. Let

$$
T_{n}:=\inf \left\{k \in \mathbf{N}: R_{k}^{(n)}=n-1\right\}=\sum_{l=1}^{\infty} 1_{\left\{R_{l}^{(n)}<n-1\right\}}+1
$$

be the absorption time. Note that $T_{1}=1$ almost surely, while $T_{n}<\infty$ almost surely for $n \geq 2$ (see Lemma 2.1).

Below we provide some examples of applications of random walks $\left\{R_{k}^{(n)}: k \in \mathbf{N}\right\}$ with a barrier.

Example 1. Let $\left\{Z_{k}: k \in \mathbf{N}\right\}$ be a death chain with the space of states $\mathbf{N}$. Let the transition probabilities be such that $\pi_{i j}>0$ for $j<i$ and $\pi_{i j}=0$ otherwise. Define the random variables

$$
X_{n}:=\inf \left\{k \geq 1: Z_{k}=1 \text { given } Z_{0}=n\right\},
$$

which are called the absorption times of the death chain. It is shown in the paper 8 ] that if

$$
\pi_{n, n-k}=\frac{\mathrm{P}\{\xi=k\}}{\mathrm{P}\{\xi<n-1\}}
$$

2000 Mathematics Subject Classification. Primary 60J80, 60E99; Secondary 60G42.

Key words and phrases. Random walks with a barrier, absorption moments.

The research is supported by DFG, project 436UKR 113/93/0-1. 
then the distribution of $X_{n}$ for natural numbers $n \geq 1+\sup \{k \in \mathbf{N}: \mathrm{P}\{\xi \geq k\}=1\}$ coincides with the distribution of the random variable

$$
M_{n}:=\#\left\{i \in \mathbf{N}: R_{i-1}^{(n)} \neq R_{i}^{(n)}\right\}=\sum_{l=0}^{\infty} 1_{\left\{R_{l}^{(n)}+\xi_{l+1}<n\right\}} .
$$

By $Y_{n}$, we denote the number of deleted edges in the procedure of separating the root of a random recursive tree with $n$ vertices (this procedure is proposed in [10). It is shown in the paper [9] that if

$$
\mathrm{P}\{\xi=k\}:=\frac{1}{k(k+1)}, \quad k \in \mathbf{N},
$$

then

$$
Y_{n} \stackrel{\mathrm{d}}{\rightarrow} X_{n} \stackrel{\mathrm{d}}{\rightarrow} M_{n}, \quad n \in \mathbf{N} .
$$

Example 2. Assume that the wage fund of an employer is 50,000 units of a currency per month. We also assume that the salary demands of job applicants are independent copies of a random variable $\xi$ assuming values in multiples of 500 units. Then the random variable $M_{50001}$ means the number of employees, while the random variable $T_{50001}$ is the total number of seekers trying to get a job at this business.

Some further applications of random walks with a barrier are also considered in the paper [6].

In what follows, a law $\mu_{\alpha}, \alpha \in[1,2)$, is called stable if its characteristic function is of the following form:

$$
\begin{cases}\exp \left\{-|t|^{\alpha} \Gamma(1-\alpha)(\cos (\pi \alpha / 2)+i \sin (\pi \alpha / 2) \operatorname{sgn}(t))\right\}, & 1<\alpha<2, \\ \exp \{-|t|(\pi / 2-i \ln |t| \operatorname{sgn}(t))\}, & \alpha=1 .\end{cases}
$$

Now we provide the main results of the paper concerning the asymptotic behavior of the sequence $\left\{T_{n}: n \in \mathbf{N}\right\}$ as $n \rightarrow \infty$.

Theorem 1.1. If $m:=\mathrm{E} \xi<\infty$, then the following statements are equivalent.

(i) There are sequences $\{a(n), b(n): n \in \mathbf{N}\}, a(n)>0$ and $b(n) \in \mathbf{R}$ such that $\left(T_{n}-b(n)\right) / a(n)$ weakly converges as $n \rightarrow \infty$ to a nondegenerate and proper probability distribution.

(ii) For some $\alpha \in[1,2]$ and for some slowly varying at $\infty$ function $L$,

$$
\sum_{k=1}^{n} k^{2} p_{k} \sim n^{2-\alpha} L(n), \quad n \rightarrow \infty .
$$

If $\sigma^{2}:=\operatorname{Var} \xi<\infty$, then one can choose $b(n):=n / m$ and $a(n):=\left(m^{-3} \sigma^{2} n\right)^{1 / 2}$; the limit distribution in this case is normal with zero mean and variance 1 .

If $\sigma^{2}=\infty$ and (11) holds for $\alpha=2$, then one can choose $b(n):=n / m$ and $a(n):=$ $c(n) m^{-3 / 2}$, where the nonnegative sequence $c(n)$ is such that $\lim _{n \rightarrow \infty} n L(c(n)) / c^{2}(n)$ $=1$. The limit distribution in this case is normal with zero mean and variance 1 .

If (1) holds for $\alpha \in[1,2)$, then one can choose $b(n):=n / m$ and $a(n):=c(n) / m^{(\alpha+1) / \alpha}$, where $c(n)$ is a nonnegative sequence such that

$$
\lim _{n \rightarrow \infty} n L(c(n)) / c^{\alpha}(n)=\frac{2-\alpha}{\alpha} .
$$

The limit distribution in this case is $\mu_{\alpha}$. 
Theorem 1.2. Let, for $\alpha \in(0,1)$,

$$
\sum_{k=n}^{\infty} p_{k} \sim n^{-\alpha} L(n), \quad n \rightarrow \infty,
$$

where $L$ is a slowly varying at $\infty$ function. Then

$$
\frac{T_{n}}{a(n)} \stackrel{d}{\rightarrow} \int_{0}^{\infty} e^{-U(t)} d t, \quad n \rightarrow \infty,
$$

where $a(n):=n^{\alpha} L^{-1}(n)$ and where $\{U(t): t \geq 0\}$ is the subordinator with zero shift and Lévy measure

$$
\nu(d t)=\frac{e^{-t / \alpha}}{\left(1-e^{-t / \alpha}\right)^{\alpha+1}} d t, \quad t>0 .
$$

Theorem 1.3. Let $\mathrm{E} \xi=\infty$ and

$$
\sum_{k=n}^{\infty} p_{k} \sim L(n) / n, \quad n \rightarrow \infty
$$

for some slowly varying at $\infty$ function $L$. Let $c(t)$ be an arbitrary positive function such that $\lim _{t \rightarrow \infty} t L(c(t)) / c(t)=1$. Put $\psi(t):=t \int_{0}^{c(t)} \mathrm{P}\{\xi>y\} d y$. Let $b(t)$ be another arbitrary positive function such that

$$
b(\psi(t)) \sim \psi(b(t)) \sim t, \quad t \rightarrow \infty .
$$

Put $a(t):=t^{-1} b(t) c(b(t))$. Then $\left(T_{n}-b(n)\right) / a(n)$ weakly converges to the stable law $\mu_{1}$.

It is shown in the paper [8] that Theorems 1.1, 1.2, and 1.3 hold if $T_{n}$ is changed for $M_{n}$. The results of our paper do not follow from results of [8]; however, our proofs are similar to those in 8 .

\section{Auxiliary Results}

For all $m \in \mathbf{N}$ and all $i \in \mathbf{N}$, let $\widehat{R}_{0}^{(m)}(i):=0$,

$$
\widehat{R}_{k}^{(m)}(i):=\widehat{R}_{k-1}^{(m)}(i)+\xi_{i+k} 1_{\left\{\widehat{R}_{k-1}^{(m)}(i)+\xi_{i+k}<m\right\}}, \quad k \in \mathbf{N},
$$

and

$$
\widehat{T}_{m}(i):=\sum_{l=1}^{\infty} 1_{\left\{\widehat{R}_{l}^{(m)}(i)<m-1\right\}}+1
$$

Note that the distributions of the random variables $\widehat{T}_{m}(i)$ and $T_{m}$ are identical. Put $\widehat{T} .:=\widehat{T} .(1)$.

Lemma 2.1. The random variable $T_{n}$ is finite almost surely for all finite $n \in \mathbf{N}$.

Proof. We use the induction in $n$. It is clear that $T_{1}=1$ almost surely. Let $T_{k}<\infty$ almost surely for $k=1,2, \ldots, n-1$. We show that $T_{n}<\infty$ almost surely, too.

The sequence $\left\{\widehat{R}_{k}^{(n)}(1) \in \mathbf{N}\right\}$ as well as the sequence $\widehat{T}_{n}$ does not depend on $\xi_{1}$. Moreover,

$$
T_{n}=\left(1+\widehat{T}_{n-\xi_{1}}(1)\right) 1_{\left\{\xi_{1} \leq n-2\right\}}+1_{\left\{\xi_{1}=n-1\right\}}+\left(1+\widehat{T}_{n}(1)\right) 1_{\left\{\xi_{1} \geq n\right\}} .
$$

By the induction assumption, $\widehat{T}_{k}<\infty, k=1,2, \ldots, n-1$. Thus

$$
\widehat{T}_{n-\xi_{1}} 1_{\left\{\xi_{1} \leq n-2\right\}}=\sum_{k=1}^{n-2} \widehat{T}_{n-k} 1_{\left\{\xi_{1}=k\right\}}<\infty .
$$


Therefore

$$
\mathrm{P}\left\{T_{n}=\infty\right\}=\mathrm{P}\left\{\left(1+\widehat{T}_{n}\right) 1_{\left\{\xi_{1} \geq n\right\}}=\infty\right\}=\mathrm{P}\left\{T_{n}=\infty\right\} \mathrm{P}\left\{\xi_{1} \geq n\right\},
$$

whence

$$
\mathrm{P}\left\{T_{n}=\infty\right\}=0,
$$

since $\mathrm{P}\left\{\xi_{1} \geq n\right\}<1$. The lemma is proved.

Put $S_{0}:=0$ and $S_{n}:=\xi_{1}+\cdots+\xi_{n}$ for $n \in \mathbf{N}$. Let

$$
N_{n}:=\inf \left\{k \geq 1: S_{k} \geq n\right\} .
$$

We show that the sequences $T_{n}, n \in \mathbf{N}$, and $\mathrm{E} T_{n}, n \in \mathbf{N}$, satisfy certain recurrence relations.

Proposition 2.1. For all $n \in \mathbf{N}$,

$$
T_{n}=N_{n}+\widehat{T}_{Y_{n}}\left(N_{n}\right)-2 \cdot 1_{\left\{Y_{n}=1\right\}}
$$

with probability one, where $Y_{n}:=n-S_{N_{n}-1}$.

Proof. We have

$$
\begin{aligned}
T_{n}= & T_{n}\left(1_{\left\{Y_{n}=1\right\}}+1_{\left\{Y_{n} \neq 1\right\}}\right)=\left(N_{n}-1\right) 1_{\left\{Y_{n}=1\right\}}+T_{n} 1_{\left\{Y_{n} \neq 1\right\}} \\
= & \left(N_{n}-1\right) 1_{\left\{Y_{n}=1\right\}}+\sum_{k=1}^{N_{n}-1} 1_{\left\{R_{k}^{(n)}<n-1\right\}} 1_{\left\{Y_{n} \neq 1\right\}}+\sum_{k=N_{n}+1}^{\infty} 1_{\left\{R_{k}^{(n)}<n-1\right\}} 1_{\left\{Y_{n} \neq 1\right\}} \\
& +2 \cdot 1_{\left\{Y_{n} \neq 1\right\}} \\
= & N_{n}-1+2 \cdot 1_{\left\{Y_{n} \neq 1\right\}}+\sum_{k=N_{n}+1}^{\infty} 1_{\left\{R_{k}^{(n)}<n-1\right\}} 1_{\left\{Y_{n} \neq 1\right\}} .
\end{aligned}
$$

If the random event $\left\{Y_{n}=1, k \geq N_{n}+1\right\}$ occurs, then $R_{k}^{(n)}=n-1$. Thus

$$
\sum_{k=N_{n}+1}^{\infty} 1_{\left\{R_{k}^{(n)}<n-1\right\}} 1_{\left\{Y_{n} \neq 1\right\}}=\sum_{k=N_{n}+1}^{\infty} 1_{\left\{R_{k}^{(n)}<n-1\right\}} .
$$

Therefore,

$$
\begin{aligned}
T_{n} & =N_{n}-1+2 \cdot 1_{\left\{Y_{n} \neq 1\right\}}+\sum_{k=N_{n}+1}^{\infty} 1_{\left\{R_{k}^{(n)}<n-1\right\}} \\
& =N_{n}-1+2 \cdot 1_{\left\{Y_{n} \neq 1\right\}}+\sum_{k=1}^{\infty} 1_{\left\{\widehat{R}_{k}^{\left(n-S_{N_{n}-1}\right)}\left(N_{n}\right)<n-S_{N_{n}-1}-1\right\}} \\
& =N_{n}+\widehat{T}_{n-S_{N_{n}-1}}\left(N_{n}\right)-2 \cdot 1_{\left\{Y_{n}=1\right\}},
\end{aligned}
$$

and this completes the proof of Proposition 2.1.

Proposition 2.2. For all $n \in \mathbf{N}$ and $k \in \mathbf{N}$,

$$
\mathrm{E} T_{n}^{k}=r_{n} \sum_{l=1}^{n-1} \mathrm{E} T_{n-l}^{k} p_{l}+k \cdot r_{n} \mathrm{E} T_{n}^{k-1}+D_{k}\left(\mathrm{E} T_{n}, \mathrm{E} T_{n}^{2}, \ldots, \mathrm{E} T_{n}^{k-2}\right),
$$

where

$$
r_{n}:=\frac{1}{p_{1}+p_{2}+\cdots+p_{n-1}}, \quad \text { D. }\left(x_{1}, x_{2}, \ldots, x_{n}\right):=v^{(0)}+\sum_{k=1}^{n} v^{(k)} x_{k},
$$

and $\left\{v^{(k)}, k=0,1,2, \ldots, n\right\}$ are some real numbers. 
In particular, if $k=1$, then

$$
\mathrm{E} T_{n}=r_{n} \mathrm{P}\left\{\xi_{1} \neq n-1\right\}+r_{n} \sum_{k=1}^{n-1} \mathrm{E} T_{n-k} p_{k} .
$$

Proof. We have

$$
\begin{aligned}
\mathrm{P}\left\{T_{n}=i\right\}= & \sum_{l=1}^{\infty} \mathrm{P}\left\{T_{n}=i, \xi_{1}=l\right\} \\
= & \sum_{l=1}^{n-2} \mathrm{P}\left\{T_{n}=i, \xi_{1}=l\right\}+\mathrm{P}\left\{T_{n}=i, \xi_{1}=n-1\right\}+\mathrm{P}\left\{T_{n}=i, \xi_{1} \geq n\right\} \\
= & \sum_{l=1}^{n-1} \mathrm{P}\left\{T_{n-l}=i-1\right\} \mathrm{P}\left\{\xi_{1}=l\right\}-1_{\{i=2\}} \mathrm{P}\left\{\xi_{1}=n-1\right\} \\
& +1_{\{i=1\}} \mathrm{P}\left\{\xi_{1}=n-1\right\}+\mathrm{P}\left\{T_{n}=i-1\right\} \mathrm{P}\left\{\xi_{1} \geq n\right\}
\end{aligned}
$$

Then

$$
\begin{aligned}
\mathrm{E} T_{n}^{k}= & \sum_{i=0}^{\infty} i^{k} \mathrm{P}\left\{T_{n}=i\right\} \\
=\sum_{i=0}^{\infty} i^{k}\left[\sum_{l=1}^{n-1} \mathrm{P}\left\{T_{n-l}=i-1\right\} \mathrm{P}\left\{\xi_{1}=l\right\}-1_{\{i=2\}} \mathrm{P}\left\{\xi_{1}=n-1\right\}\right. & \\
& \left.\quad+1_{\{i=1\}} \mathrm{P}\left\{\xi_{1}=n-1\right\}+\mathrm{P}\left\{T_{n}=i-1\right\} \mathrm{P}\left\{\xi_{1} \geq n\right\}\right] .
\end{aligned}
$$

Further

$$
\begin{gathered}
\sum_{i=0}^{\infty}(i-1+1)^{k} \sum_{l=1}^{n-1} \mathrm{P}\left\{T_{n-l}=i-1\right\} \mathrm{P}\left\{\xi_{1}=l\right\}=\sum_{l=1}^{n-1} \mathrm{E}\left(T_{n-l}\right)^{k} p_{l}+\sum_{j=1}^{n-1}\left(\begin{array}{l}
k \\
j
\end{array}\right) \mathrm{E} T_{n-l}^{j}+1, \\
\sum_{i=0}^{\infty} i^{k} \mathrm{P}\left\{T_{n}=k-1\right\} \mathrm{P}\left\{\xi_{1} \geq n\right\}=\left(\mathrm{E}\left(T_{n}\right)^{k}+\sum_{j=1}^{n-1}\left(\begin{array}{c}
k \\
j
\end{array}\right) \mathrm{E} T_{n}^{j}+1\right) \sum_{l=n}^{\infty} p_{l},
\end{gathered}
$$

whence

$\mathrm{E} T_{n}^{k}=r_{n} \sum_{l=1}^{n-1} \mathrm{E} T_{n-l}^{k} p_{l}+k r_{n}\left[\sum_{l=1}^{n-1} \mathrm{E} T_{n-l}^{k-1} p_{l}+\mathrm{E} T_{n}^{k-1} \sum_{l=n}^{\infty} p_{l}\right]+D_{k_{1}}\left(\mathrm{E} T_{n}, \mathrm{E} T_{n}^{2}, \ldots, \mathrm{E} T_{n}^{k-2}\right)$.

Using the latter relation for the moment $\mathrm{E} T_{n}^{k-1}$, we complete the proof of (6).

Lemma 2.2. Let condition (2) hold for $\alpha \in(0,1)$. Then

$$
\lim _{n \rightarrow \infty} \frac{L(n)}{n^{\alpha}} \mathrm{E} T_{n}=\frac{\Gamma(1-\alpha) \Gamma(1+\alpha)}{\Gamma(1-\alpha) \Gamma(1+\alpha)-1} .
$$

Proof. It is known that

$$
\lim _{n \rightarrow \infty} \frac{L(n)}{n^{\alpha}} \mathrm{E} N_{n}=\frac{1}{\Gamma(1-\alpha) \Gamma(1+\alpha)}
$$

(see, for example, [8]). Put

$$
b:=\frac{\Gamma(1-\alpha) \Gamma(1+\alpha)}{\Gamma(1-\alpha) \Gamma(1+\alpha)-1}
$$


We show that

$$
\varlimsup_{n \rightarrow \infty} \frac{\mathrm{E} T_{n}}{\mathrm{E} N_{n}} \leq b .
$$

Assume the converse. Then, for an arbitrary $\varepsilon>0$, the inequality $\mathrm{E} T_{n}>(b+\varepsilon) \mathrm{E} N_{n}$ holds for infinitely many numbers $n$. Thus one can choose a positive number $\varepsilon$ such that $\mathrm{E} T_{n}>(b+\varepsilon) \mathrm{E} N_{n}+c$ infinitely often for any fixed $c>0$. Let

$$
n_{c}:=\inf \left\{n \geq 1: \mathrm{E} T_{n}>(b+\varepsilon) \mathrm{E} N_{n}+c\right\} .
$$

Note that $\lim _{c \rightarrow \infty} n_{c}=\infty$. Then

$$
\mathrm{E} T_{n} \leq(b+\varepsilon) \mathrm{E} N_{n}+c, \quad n \in\left\{1,2, \ldots, n_{c}-1\right\} .
$$

Therefore

$$
\begin{aligned}
(b+\varepsilon) \mathrm{E} N_{n_{c}}+c<\mathrm{E} T_{n_{c}} & \stackrel{(7)}{=} r_{n_{c}} \mathrm{P}\left\{\xi_{1} \neq n_{c}-1\right\}+r_{n_{c}} \sum_{k=1}^{n_{c}-1} \mathrm{E} T_{n_{c}-k} p_{k} \\
& \leq r_{n_{c}} \mathrm{P}\left\{\xi_{1} \neq n_{c}-1\right\}+c+r_{n_{c}}(b+\varepsilon) \sum_{k=1}^{n_{c}-1} \mathrm{E} N_{n_{c}-k} p_{k} .
\end{aligned}
$$

Lemma 3.4 of 8 implies that $\mathrm{E} N_{n}$ is such that

$$
\mathrm{E} N_{n}=1+\sum_{k=1}^{n-1} \mathrm{E} N_{n-k} p_{k} \text {. }
$$

Hence

$$
(b+\varepsilon) \mathrm{E} N_{n_{c}}+c<r_{n_{c}} \mathrm{P}\left\{\xi_{1} \neq n_{c}-1\right\}+c+r_{n_{c}}(b+\varepsilon)\left(\mathrm{E} N_{n_{c}}-1\right)
$$

or, equivalently,

$$
0<r_{n_{c}} \mathrm{P}\left\{\xi \neq n_{c}-1\right\}-r_{n_{c}}(b+\varepsilon)+\mathrm{E} N_{n_{c}}\left(r_{n_{c}}-1\right)(b+\varepsilon) .
$$

Since $r_{n}-1 \sim n^{-\alpha} L(n), n \rightarrow \infty$, we pass to the limit in the latter inequality as $c \rightarrow \infty$ and obtain

$$
\frac{\varepsilon}{b}<b-1+1-b=0 .
$$

This contradiction proves (8). Similar reasoning shows that the converse inequality holds for the lower limit as well. This proves the lemma.

Theorem 2.3. If

$$
\sum_{l=1}^{n} \sum_{k=l}^{\infty} p_{k} \sim L(n), \quad n \rightarrow \infty,
$$

for some slowly varying at $\infty$ function $L$, then

$$
\frac{T_{n}}{\mathrm{E} T_{n}} \stackrel{\mathrm{P}}{\rightarrow} 1
$$

Moreover $\mathrm{E} T_{n} \sim n / L(n)$ as $n \rightarrow \infty$.

Proof. To prove the theorem, it is sufficient to show that

$$
\lim _{n \rightarrow \infty} \frac{\mathrm{E} T_{n}^{k}}{\mathrm{E} N_{n}^{k}}=1
$$

for all $k \in \mathbf{N}$, since $\mathrm{E} N_{n}^{k} \sim n^{k} / L^{k}(n)$ according to Remark 3.5 in [8].

We prove relation (11) by induction in $k$. Assume that it holds for $k \in\{1,2, \ldots, m-1\}$, but, at the same time,

$$
\varlimsup_{n \rightarrow \infty} \frac{\mathrm{E} T_{n}^{k}}{\mathrm{E} N_{n}^{k}}>1 .
$$


Then, for an arbitrary $\varepsilon>0$, the inequality $\mathrm{E} T_{n}^{k}>(\varepsilon+1) \mathrm{E} N_{n}^{k}$ holds for infinitely many numbers $n$. Then one can choose $\varepsilon$ such that $\mathrm{E} T_{n}^{k}>(\varepsilon+1) \mathrm{E} N_{n}^{k}+c$ infinitely often for any fixed $c>0$. Put

$$
n_{c}:=\inf \left\{n \geq 1: \mathrm{E} T_{n}^{k}>(\varepsilon+1) \mathrm{E} N_{n}^{k}+c\right\} .
$$

Note that $\lim _{c \rightarrow \infty} n_{c}=\infty$. Then

$$
\mathrm{E} T_{n}^{k} \leq(1+\varepsilon) \mathrm{E} N_{n}^{k}+c, \quad n \in\left\{1,2, \ldots, n_{c}-1\right\} .
$$

According to Lemma 3.4 of $\left[8\right.$, the absorption times of the random variable $N_{n}$ satisfy the relation

$$
\mathrm{E} N_{n}^{k}=D_{k}\left(\mathrm{E} N_{n}, \ldots, \mathrm{E} N_{n}^{k-2}\right)+k \mathrm{E} N_{n}^{k-1}+\sum_{i=1}^{n-1} \mathrm{E} N_{n-i}^{k} p_{i}
$$

Thus

$$
\begin{aligned}
(1+\varepsilon) & \mathrm{E} N_{n_{c}}^{k}+c<\mathrm{E} T_{n_{c}}^{k} \stackrel{\sqrt[6]{=}}{=} r_{n} \sum_{l=1}^{n_{c}-1} \mathrm{E} T_{n_{c}-l}^{k} p_{l}+k \cdot r_{n} \mathrm{E} T_{n_{c}}^{k-1}+D_{k}\left(\mathrm{E} T_{n_{c}}, \ldots, \mathrm{E} T_{n_{c}}^{k-2}\right) \\
& \stackrel{(12)}{\leq} r_{n}(1+\varepsilon) \sum_{l=1}^{n_{c}-1} \mathrm{E} N_{n_{c}-l}^{k} p_{l}+k \cdot r_{n} \mathrm{E} T_{n_{c}}^{k-1}+D_{k}\left(\mathrm{E} T_{n_{c}}, \ldots, \mathrm{E} T_{n_{c}}^{k-2}\right)+c \\
= & (1+\varepsilon)\left(r_{n_{c}}-1\right)\left(\mathrm{E} N_{n_{c}}^{k}-D_{k}\left(\mathrm{E} N_{n_{c}}\right)-k \mathrm{E} N_{n_{c}}^{k-1}\right)+(1+\varepsilon) \mathrm{E} N_{n_{c}}^{k} \\
& -(1+\varepsilon)\left(D_{k}\left(\mathrm{E} N_{n_{c}}\right)+k \mathrm{E} N_{n_{c}}^{k-1}\right)+k \cdot r_{n} \mathrm{E} T_{n_{c}}^{k-1}+D_{k}\left(\mathrm{E} T_{n_{c}}\right)+c,
\end{aligned}
$$

where $D_{k}(\mathrm{E} X):=D_{k}\left(\mathrm{E} X, \mathrm{E} X^{2}, \ldots, \mathrm{E} X^{k-2}\right)$. Therefore

$$
\begin{aligned}
0< & (1+\varepsilon)\left(r_{n_{c}}-1\right)\left(\mathrm{E} N_{n_{c}}^{k}-D_{k}\left(\mathrm{E} N_{n_{c}}\right)-k \mathrm{E} N_{n_{c}}^{k-1}\right)-(1+\varepsilon)\left(D_{k}\left(\mathrm{E} N_{n_{c}}\right)+k \mathrm{E} N_{n_{c}}^{k-1}\right) \\
& +k r_{n} \mathrm{E} T_{n_{c}}^{k-1}+D_{k}\left(\mathrm{E} T_{n_{c}}\right) .
\end{aligned}
$$

We divide the latter inequality by $\mathrm{E} N_{n_{c}}^{k-1}$ and pass to the limit as $c \rightarrow \infty$. In view of Remark 3.5 of [8], we have $\mathrm{E} N_{n}^{k-1} \sim n^{k-1} / L^{k-1}(n)$. Condition (9) and Theorem 1.7.2 of [2] imply that $\lim _{n \rightarrow \infty}\left(r_{n}-1\right) n / L(n)=0$. By the assumption of the induction,

$$
\lim _{c \rightarrow \infty} \frac{D_{k}\left(\mathrm{E} T_{n_{c}}, \ldots, \mathrm{E} T_{n_{c}}^{k-2}\right)}{\mathrm{E} N_{n}^{k-1}}=0 \text { and } \lim _{c \rightarrow \infty} \frac{\mathrm{E} T_{n_{c}}^{k-1}}{\mathrm{E} N_{n_{c}}^{k-1}}=1 \text {, }
$$

whence

$$
0<k-(1+\varepsilon) k=-\varepsilon k .
$$

This contradiction proves that

$$
\varlimsup_{n \rightarrow \infty} \frac{\mathrm{E} T_{n}^{k}}{\mathrm{E} N_{n}^{k}} \leq 1 .
$$

The inequality for the lower limit is proved similarly. Thus (11) holds for all $k \in \mathbf{N}$. The theorem is proved.

Remark 2.1. One can see from the proof of Theorem 2.3 that

$$
\frac{T_{n}}{N_{n}} \stackrel{\mathrm{P}}{\rightarrow} 1, \quad n \rightarrow \infty,
$$

provided condition (9) holds. 


\section{Proof of Theorem 1.1}

It is known that

$$
Y_{n} \stackrel{d}{\rightarrow} Y \quad \text { as } n \rightarrow \infty
$$

if $m:=\mathrm{E} \xi<\infty$ (see, for example, [8]), where the distribution of the random variable $Y$ is $\mathrm{P}\{Y=k\}=m^{-1} \mathrm{P}\{\xi \geq k\}$. Thus we obtain from (5) and (15) that

$$
T_{n}-N_{n} \stackrel{\mathrm{d}}{\rightarrow} T_{Y}^{\prime}-2 \cdot 1_{\{Y=1\}}, \quad n \rightarrow \infty .
$$

Considering an arbitrary sequence $d(n)$ such that $\lim _{n \rightarrow \infty} d(n)=\infty$, we get

$$
\frac{T_{n}-N_{n}}{d(n)} \stackrel{\mathrm{P}}{\rightarrow} 0, \quad n \rightarrow \infty .
$$

Assume that the distribution of $\xi$ does not belong to the domain of attraction of any stable law with the index $\alpha \in[1,2]$. Then there are no subsequences $x(n) \in \mathbf{R}$ and $y(n)>0$ such that $\left(S_{n}-x(n)\right) / y(n)$ converges weakly to a nondegenerate proper probability law. Since

$$
\mathrm{P}\left\{N_{n}>m\right\}=\mathrm{P}\left\{S_{m} \leq n-1\right\},
$$

the same property holds for $N_{n}$, too.

On the other hand, assume that (1) holds and let $\alpha \in[1,2)$. Then

$$
\sum_{k=n}^{\infty} p_{k} \sim \frac{\alpha}{2-\alpha} n^{-\alpha} L(n) .
$$

According to a theorem in the paper [4,

$$
\frac{N_{n}-b(n)}{a(n)} \Rightarrow \mu_{\alpha}, \quad n \rightarrow \infty,
$$

where $a(n)$ and $b(n)$ are as defined in [4].

If $\alpha=2$, then there exists a sequence of nonrandom numbers $a_{n}$ such that

$$
n a_{n}^{-2} L\left(a_{n}\right) \rightarrow 1 \quad \text { as } n \rightarrow \infty
$$

and

$$
\lim _{n \rightarrow \infty} \mathrm{P}\left\{\frac{S_{n}-n m}{a_{n}} \leq x\right\}=\mu_{2}(-\infty, x],
$$

where $\mu_{2}$ is the standard normal distribution (see, for example, [12]). Then we derive from Theorem 2 of 5 that

$$
\frac{N_{n}-b(n)}{a(n)} \Rightarrow \mu_{2} .
$$

\section{Proof of Theorem 1.2}

Repeating the reasoning of the proof of Theorem 1.4 in [8], one can show that the sequence $T_{n} / a(n)$ converges in distribution to some random variable $T$. Put

$$
Y_{n}:=n-S_{N_{n}-1} .
$$

Then

$$
\frac{T_{n}}{a(n)}=\frac{\widehat{T}_{Y_{n}}\left(N_{n}\right)}{a\left(Y_{n}\right)} \frac{a\left(Y_{n}\right)}{a(n)}+\frac{N_{n}-2 \cdot 1_{\left\{Y_{n}=1\right\}}}{a(n)} .
$$


Let $T$. be the absorption time for the sequence $\left\{\left(R_{l}^{(\cdot)}\right)^{\prime}: l \in \mathbf{N}_{0}\right\}$, which is an independent copy of $\left\{R_{l}^{(\cdot)}, l \in \mathbf{N}_{0}\right\}$ and which is independent of $\left(N_{n}, Y_{n}\right)$. For every fixed number $m \in \mathbf{N}_{0}$,

$$
\begin{aligned}
\mathrm{P}\left\{\widehat{T}_{Y_{n}}\left(N_{n}\right)=m\right\}=\sum_{i=1}^{n} \sum_{j=0}^{n-1} \mathrm{P}\left\{\widehat{T}_{n-j}(i)=m, N_{n}=i, S_{N_{n}-1}=j\right\} \\
=\sum_{i=1}^{n} \sum_{j=0}^{n-1} \mathrm{P}\left\{\sum_{l=0}^{\infty} 1_{\left\{\widehat{R}_{l}^{(n-j)}(i)<n-j-1\right\}}=m-1, N_{n}=i, S_{N_{n}-1}=j\right\} \\
=\sum_{i=1}^{n} \sum_{j=0}^{n-1} \mathrm{P}\left\{\sum_{l=0}^{\infty} 1_{\left\{\widehat{R}_{l}^{(n-j)}(i)<n-j-1\right\}}=m-1\right\} \mathrm{P}\left\{N_{n}=i, S_{N_{n}-1}=j\right\} \\
=\sum_{i=1}^{n} \sum_{j=0}^{n-1} \mathrm{P}\left\{\sum_{l=0}^{\infty} 1_{\left\{\left(R_{l}^{(n-j)}\right)^{\prime}<n-j-1\right\}}=m-1\right\} \mathrm{P}\left\{N_{n}=i, S_{N_{n}-1}=j\right\} \\
=\sum_{i=1}^{n} \sum_{j=0}^{n-1} \mathrm{P}\left\{T_{n-j}^{\prime}=m\right\} \mathrm{P}\left\{N_{n}=i, S_{N_{n}-1}=j\right\}=\mathrm{P}\left\{T_{Y_{n}}^{\prime}=m\right\} .
\end{aligned}
$$

This proves the identity of the distributions, namely

$$
\widehat{T}_{Y_{n}}\left(N_{n}\right) \stackrel{\mathrm{d}}{=} T_{Y_{n}}^{\prime}
$$

where the sequence $\left\{T_{n}^{\prime}: n \in \mathbf{N}\right\}$ does not depend on $\left(N_{n}, n-S_{N_{n}-1}\right)$ and has the same distribution as

$$
\left\{T_{n}: n \in \mathbf{N}\right\} .
$$

Since $Y_{n} \stackrel{\mathrm{P}}{\rightarrow} \infty$, we have

$$
\frac{T_{Y_{n}}^{\prime}}{a\left(Y_{n}\right)} \stackrel{\mathrm{P}}{\rightarrow} T^{\prime}
$$

where $T^{\prime}$ has the same distribution as $T$. Proposition 3.9 of 8 implies that

$$
\left(\frac{a\left(Y_{n}\right)}{a(n)}, \frac{N_{n}}{a(n)}\right) \stackrel{\mathrm{d}}{\rightarrow}\left(e^{-U(V)}, \int_{0}^{V} e^{-U(t)} d t\right), \quad n \rightarrow \infty,
$$

where $V$ is a random variable with the density $e^{-x} 1_{\{x>0\}}$ that does not depend on the subordinator $U(t)$. Put $X:=\exp \{-U(V)\}$ and $Y:=\int_{0}^{V} e^{-U(t)} d t$. Since the left hand side of (14) weakly converges, the random variables

$$
\xi_{n}:=\left(\frac{T_{Y_{n}}^{\prime}}{a\left(Y_{n}\right)}, \frac{a\left(Y_{n}\right)}{a(n)}, \frac{N_{n}-2 \cdot 1_{\left\{Y_{n}=1\right\}}}{a(n)}\right)
$$

weakly converge, too. Taking into account (14) and (17) we prove that

$$
\xi_{n} \stackrel{\mathrm{d}}{\rightarrow}\left(T^{\prime}, X, Y\right) .
$$

Passing to the limit as $n \rightarrow \infty$ we prove that

$$
T \stackrel{\mathrm{d}}{\rightarrow} X T^{\prime}+Y,
$$

where $T^{\prime}$ does not depend on the pair $(X, Y)$. To prove this relation, it suffices to show that

$$
\lim _{n \rightarrow \infty}\left|\mathrm{E} \exp \left\{i\left(t \frac{T_{Y_{n}}^{\prime}}{a\left(Y_{n}\right)}+v \frac{a_{Y_{n}}}{a(n)}+u \frac{N_{n}-2 \cdot 1_{\left\{Y_{n}=1\right\}}}{a(n)}\right)\right\}-\mathrm{E} e^{i t T^{\prime}} \mathrm{E} e^{i(v X+u Y)}\right|=0
$$

for all real $t, u$, and $v$. 
In view of (16) and (17) and since $Y_{n} \rightarrow \infty$ as $n \rightarrow \infty$, we obtain that, for all $\varepsilon>0$, there are $N_{i}=N_{i}(\varepsilon), i=1,2,3$, such that

$$
\begin{gathered}
\left|\mathrm{E} \exp \left\{i t \frac{T_{Y_{n}}^{\prime}}{a\left(Y_{n}\right)}\right\}-\mathrm{E} e^{i t T^{\prime}}\right|<\varepsilon, \quad n>N_{1}, \\
\left|\mathrm{E} \exp \left\{i\left(v \frac{a_{Y_{n}}}{a(n)}+u \frac{N_{n}-2 \cdot 1_{\left\{Y_{n}=1\right\}}}{a(n)}\right)\right\}-\mathrm{E} e^{i(v X+u Y)}\right|<\varepsilon, \quad n>N_{2}, \\
\mathrm{P}\left\{Y_{n} \leq \max \left\{N_{1}, N_{2}\right\}\right\}<\varepsilon, \quad n>N_{3} .
\end{gathered}
$$

Put $N:=\max \left\{N_{1}, N_{2}\right\}$. Then

$$
\begin{aligned}
\mid \mathrm{E} \exp & \left\{i\left(t \frac{T_{Y_{n}}^{\prime}}{a\left(Y_{n}\right)}+v \frac{a_{Y_{n}}}{a(n)}+u \frac{N_{n}-2 \cdot 1_{\left\{Y_{n}=1\right\}}}{a(n)}\right)\right\}-\mathrm{E} e^{i t T^{\prime}} \mathrm{E} e^{i(v X+u Y)} \mid \\
& =\mid \sum_{s=0}^{n-1} \sum_{l=1}^{s+1}\left(\mathrm{E} \exp \left\{i\left(t \frac{T_{n-s}^{\prime}}{a(n-s)}+v \frac{a_{n-s}}{a(n)}+u \frac{l-2 \cdot 1_{\{n-s=1\}}}{a(n)}\right)\right\}\right. \\
& \leq\left|\sum_{s=0}^{n-N} \sum_{l=1}^{s+1} \ldots\right|+\left|\sum_{s=n-N+1}^{n-1} \sum_{l=1}^{s+1} \ldots\right| .
\end{aligned}
$$

For all $n>\max \left(N, N_{3}\right)$, the second term is estimated as follows:

$$
\begin{aligned}
& \mid \sum_{s=n-N+1}^{n-1} \sum_{l=1}^{s+1}\left(\mathrm{E} \exp \left\{i\left(t \frac{T_{n-s}^{\prime}}{a(n-s)}+v \frac{a_{n-k}}{a(n)}+u \frac{l-2 \cdot 1_{\{n-s=1\}}}{a(n)}\right)\right\}\right. \\
& \left.\quad-\mathrm{E} e^{i t T^{\prime}} \mathrm{E} e^{i(v X+u Y)}\right) \mathrm{P}\left\{S_{l-1}=s, N_{n}=l\right\} \mid \\
& \quad \leq 2\left|\mathrm{P}\left\{Y_{n} \leq N\right\}\right|<2 \varepsilon .
\end{aligned}
$$

For the first term,

$$
\begin{aligned}
& \mid \sum_{s=0}^{n-N} \sum_{l=1}^{s+1}\left(\mathrm{E} \exp \left\{i\left(t \frac{T_{n-s}^{\prime}}{a(n-s)}+v \frac{a_{n-s}}{a(n)}+u \frac{l-2 \cdot 1_{\{n-s=1\}}}{a(n)}\right)\right\}\right. \\
& \left.-\mathrm{E} e^{i t T^{\prime}} \mathrm{E} e^{i(v X+u Y)}\right) \mathrm{P}\left\{S_{l-1}=s, N_{n}=l\right\} \mid \\
& \leq \sum_{s=0}^{n-N} \sum_{l=1}^{s+1}\left|\left(\mathrm{E} \exp \left\{i\left(t \frac{T_{n-s}^{\prime}}{a(n-s)}\right)\right\}-\mathrm{E} e^{i t T^{\prime}}\right) \mathrm{E} e^{i(v X+u Y)}\right| \mathrm{P}\left\{S_{l-1}=s, N_{n}=l\right\} \\
& +\sum_{s=0}^{n-N} \sum_{l=1}^{s+1} \mid \mathrm{E} \exp \left\{i \frac{T_{n-s}^{\prime}}{a(n-s)}\right\}\left(\mathrm{E} \exp \left\{i\left(v \frac{a_{n-s}}{a(n)}+u \frac{N_{n}-2 \cdot 1_{\{n-s=1\}}}{a(n)}\right)\right\}\right. \\
& \times \mathrm{P}\left\{S_{l-1}=s, N_{n}=l\right\}
\end{aligned}
$$


Since $T^{\prime}$ does not depend on the pair $(X, Y)$, equation (18) for the distributions has a unique solution

$$
T \stackrel{\mathrm{d}}{\rightarrow} \int_{0}^{\infty} e^{-U(t)} d t
$$

(see, for example, [1]). Thus

$$
\frac{T_{n}}{a(n)} \stackrel{\mathrm{d}}{\rightarrow} \int_{0}^{\infty} e^{-U(t)} d t, \quad n \rightarrow \infty
$$

The theorem is proved.

\section{Proof of Theorem 1.3}

Condition (3) implies that $m(x):=\int_{0}^{x} \mathrm{P}\{\xi>y\} d y, x>0$, belongs to the de Haan class $\Pi$, that is,

$$
\lim _{t \rightarrow \infty} \frac{m(\lambda t)-m(t)}{L(t)}=\ln \lambda,
$$

whence we obtain that $m(x)$ is slowly varying. Since $\sum_{l=1}^{n} \sum_{k=l}^{\infty} p_{k} \sim m(n)$, we get from Remark 2.1 that

$$
\frac{T_{n}}{N_{n}-1} \rightarrow 1, \quad n \rightarrow \infty
$$

In view of Theorem 3(c) and the formula in [1, p. 42], we deduce that

$$
\frac{N_{n}-b(n)-1}{a(n)} \Rightarrow \mu_{1} \text {. }
$$

Thus

$$
\frac{T_{n}-b(n)}{a(n)}-\frac{T_{n}-N_{n}+1}{N_{n}-1} \frac{b(n)}{a(n)} \Rightarrow \mu_{1} .
$$

Similarly to the proof of Theorem 1.5 in 8 one can show that the second term approaches zero in probability. Indeed, let

$$
\frac{T_{n}-N_{n}+1}{N_{n}-1} \frac{b(n)}{a(n)}=\frac{\widehat{T}_{Y_{n}}+1-2 \cdot 1_{\left\{Y_{n}=1\right\}}}{Y_{n} / m\left(Y_{n}\right)} \frac{m(n)}{m\left(Y_{n}\right)} \frac{b(n) Y_{n}}{n a(n)} \frac{n}{m(n)\left(N_{n}-1\right)}=: \prod_{i=1}^{4} K_{i}(n) .
$$

By Theorem $2.3 m(n) T_{n} / n \stackrel{\mathrm{P}}{\rightarrow} 1$. Using the equality of distributions (5) and that $Y_{n} \stackrel{\mathrm{P}}{\rightarrow} \infty$ as $n \rightarrow \infty$, we have $K_{1}(n) \stackrel{\mathrm{P}}{\rightarrow} 1$. Theorem 6 of [7] implies that $K_{2}(n) \stackrel{\mathrm{d}}{\rightarrow} 1 / R$, where $R$ is a random variable with the uniform distribution on $[0,1]$. Proposition 3.7 and Corollary 3.6 imply that $K_{3}(n) \stackrel{\mathrm{P}}{\rightarrow} 0$ and $K_{4}(n) \stackrel{\mathrm{P}}{\rightarrow} 1$. The theorem is proved.

\section{ACKNOWLEDGEMENT}

The author is grateful to his scientific advisor Oleksander Iksanov for suggesting the problem and for several useful comments.

\section{BIBLIOGRAPHY}

1. N. H. Bingham, Limit theorems for regenerative phenomena, recurrent events and renewal theory, Z. Wahrscheinlichkeitstheorie und Verw. Gebiete 21 (1972), 20-44. MR0353459 (50:5942)

2. N. H. Bingham, C. M. Goldie, and J. L. Teugels, Regular Variation, Cambridge University Press, Cambridge, 1989. MR1015093 (90i:26003)

3. M. Drmota, A. Iksanov, M. Möhle, and U. Rösler, A limiting distribution for the number of cuts needed to isolate the root of a random recursive tree, http://www.unicyb.kiev.ua/ iksan (2006).

4. W. Feller, Fluctuation theory of recurrent events, Trans. Amer. Math. Soc. 67 (1949), 98-119. MR0032114(11:255c) 
5. C. C. Heyde, A limit theorem for random walks with drift, J. Appl. Probab. 1 (1967), 144-150. MR.0207061 (34:6877)

6. K. Hinderer and H. Walk, Anwendung von Erneuerungstheoremen und Taubersätzen für eine Verallgemeinerung der Erneuerungsprozesse, Math. Z. 126 (1972), 95-115. MR0300354 $(45: 9400)$

7. K. B. Erickson, Strong renewal theorems with infinite mean, Trans. Amer. Math. Soc. 151 (1970), 263-291. MR0268976 (42:3873)

8. A. Iksanov and M. Möhle, On a random recursion related to absorption times of death Markov chains, http://www.unicyb.kiev.ua/ iksan (2007).

9. A. Iksanov and M. Möhle, A probabilistic proof of a weak limit law for the number of cuts needed to isolate the root of a random recursive tree, Elect. Comm. in Probab. 12 (2007), 36-43. MR2407414 (2009e:05076)

10. A. Meir and J. W. Moon, Cutting down recursive trees, Math. Biosci. 21 (1974), 173-181.

11. W. Vervaat, On a stochastic difference equation and a representation of nonnegative infinitely divisible random variables, Adv. Appl. Probab. 11 (1979), 750-783. MR.544194 (81b:60064)

12. W. Feller, An Introduction to Probability Theory and its Applications, Second edition, vol. II, John Wiley \& Sons, New York-London-Sydney, 1971. MR0270403 (42:5292)

Faculty for Cybernetics, National Taras Shevchenko University, Academician Glushkov Avenue 6, Kyiv 03127, Ukraine

E-mail address: npasha@ukr.net

Received 18/OCT/2007

Translated by S. KVASKO 